\title{
Quantitative functions for size and distance judgments
}

\author{
JOHN C. BAIRD ${ }^{1}$ AND WILLIAM R. BIERSDORF \\ WALTER REED ARMY INSTITUTE OF RESEARCH, WASHINGTON, D. C.
}

A psychophysical approach was used to obtain judgments of visual extent under three conditions. In two conditions a comparison stimulus at each of two distances was matched in size to a standard which varied in distance. Stimuli were presented on a well-lighted table and were judged by two observers under Objective instructions. Both the standard and comparison were located in either a frontal or longitudinal plane. In a third condition relative distance estimates were given of tws stimuli which varied in their relative positions along the table. The mean results for all conditions were described as a power function of physical stimulus measures. The exponent was greater than 1.0 for frontal size and usually less than 1.0 for flat size and distance. The position of the comparison affected the magnitude of the exponents to a lesser degree. These findings have relevance for interpretations of size and distance judgments.

Size-constancy refers to the event that an object of constant physical size is judged to be such, regardless of its location within the three-dimensional physical environment. In the laboratory when size judgments are based upon information received by the visual sense, perfect size-constancy seldom occurs, a result not in keeping with certain functionalist theories of perception (Gibson, 1950; Taylor, 1962). Only a particular combination of instructions, stimulus, and response factors lead to size-constancy (Baird, 1965a; Carlson, 1962; Epstein, 1963), and these results probably can be treated as special cases within a more comprehensive theory (e.g., Akishige, 1961). Before such a theory can be operational, however, several key issues need to be settled. This paper describes some progress toward these prerequisite goals.

First, we have extended the range of quantitative data collected for size and distance judgments under Objective instructions and full-cue conditions. The majority of previous results were gathered with a single method on a single value of a dimension such as relative distance or stimulus orientation. We used an extended stimulus series in three conditions. In one situation a relative-size judgment was made between a comparison stimulus at one distance and a standard stimulus at a number of different distances, when both were in a frontal plane. The second situation also involved relative size, but the stimuli were placed flat on a table extending away from the observer and beneath his primary lines of sight. In the third situation ratio estimates of distance along the table were obtained. The same two observers were used in all conditions, and the relative posi- tions of the comparison and standard were varied.

Our second concern was theoretical. Since the functionalist theory leading to size-constancy predictions is based upon metric measures of stimuli, and since this approach is inadequate when applied to most data on size and distance, we decided to explore an alternative measure-the subtended visual angle of stimuli at the eye. The task set the observer then is presumed to be the production or estimation of certain visual-angle ratios between the standard and comparison stimuli. The exact ratios depend upon the relative spatial positions of stimuli including their orientation to the eye and ground. Different stimulus and instruction variables require different ratio productions of visual angles. At present we cannot predict exactly which ratio is appropriate for each experimental condition. The important point is that the law of the visual angle is not restricted to a ratio of 1.0 in which the visual angles of the comparison and standard are equal, but is extended to include many other ratios as well.

The next step is to define quantitatively the relations among theoretical visual-angle ratios and those actually produced or estimated by observers. When metric measures (arithmetic) are used in graphic plots of theoretical and judged size, the resulting curves are not always linear. It is to our advantage if a transformation of these results would produce linear functions. A logarithmic transformation does this for judgments of size when plotted against either physical size or distance (Gogel, 1964; Stevens \& Guirao, 1963; Teghtsoonian, 1965; Ueno, 1962). We expect the same equation to hold for visual-angle functions, though the metric measures must be replaced by visual-angle measures. Such a substitution is shown in Equation 1,

$$
\phi_{\mathrm{c}}^{\prime} / \phi_{\mathrm{s}}^{\prime}=\mathrm{k}\left(\phi_{\mathrm{c}} / \phi_{\mathrm{s}}\right)^{\mathrm{n}}
$$

where $\phi_{\mathrm{c}}$ and $\phi_{\mathrm{s}}$ refer to the theoretical visual angles (in degrees) of the comparison and standard stimuli which would occur if the two were matched in physical size, $\phi_{c}^{\prime}$ and $\phi_{s}^{\prime}$ are the obtained visual angles of the comparison and standard after a size judgment, $\mathrm{k}$ is a constant dependent upon psychophysical procedure, and $\mathrm{n}$ is the exponent of the function. If the logarithm of both sides of Equation 1 is taken, we obtain Equation 2,

$$
\log \left(\phi_{c}^{\prime} / \phi_{s}^{\prime}\right)=n \log \left(\phi_{c} / \phi_{s}\right)+\log k
$$


In $\log -\log$ coordinates $\mathrm{n}$ is the slope and $\log \mathrm{k}$ the $y$-intercept of a straight line. Even though we assume $\phi_{\mathrm{s}}=\phi_{\mathrm{s}}^{\prime}$ in all our experiments, the terms are included in Equations 1 and 2 to emphasize the relative nature of size judgments. If valid, these formulas offer a convenient means for discussing the connections among size and distance data found under diverse conditions.

\section{METHOD}

A table, 18 ft. 2 in. long $\times 30$ in. wide $x 32$ in. high, was constructed. It was leveled such that no portion deviated more than $\pm 1 / 8$ in. from the horizontal The top surface was then covered with a single sheet of black photographer's cloth with no seams. The $O$ sat in a chair at one end of the table with his head positioned by a chin and forehead rest so that the front surfaces of his eyes were at the end of the table and 18 in. above the surface. The sides and far end of the table were covered by opaque cloth sheets, solid green in color, stretching upright from the surface to a height of $40 \mathrm{in}$. Good illumination of the surface was provided by fluorescent lamps on the ceiling of the room.

The stimuli for the frontal-size and flat-size experiments were cut from matte-white poster cardboard in $1 / 2$ in. widths. The standard stimuli were of $8 \mathrm{in}$. length, while the comparison series varied from $3-1 / 2$ in. to $12 \mathrm{in.} \mathrm{lengths} \mathrm{in} 1 / 4 \mathrm{in}$. steps. The frontal-size stimuli were supported on the table by small right-angle brackets cemented to the back. These supports were not visible to $O$. Delimiting stimuli for the distance estimation experiment were discs of white paper of four sizes ranging from $1 / 4$ in. to $5 / 8$ in. diameter. Illumination of all stimuli was at a daytime level of $20 \mathrm{ft} .-\mathrm{L}$ as measured with a Macbeth illuminometer.

Stimulus distances were measured from O's end of the table and were identified by tapes, invisible to $O$. A thin, black string was stretched down the center of the table from $O$ to the far end. To position a stimulus, $E$ raised the cloth sheet at the side, applied a draftman's T-square to the distance tape, and placed the stimulus in the center of the table at that distance. During this operation $O$ was asked to close his eyes, and thus, $\mathrm{E}$ was not seen by $\mathrm{O}$ at any time during the experiment. The distances of the standard and comparison stimuli for the three conditions are given in Tables 1,3 and 4.

For the frontal-size and flat experiments, run in separate series, the comparison was either at the nearest or the farthest distance. Each standard was matched in size (length) with the comparison in a method of limits, both ascending and descending. A total of eight size matches was obtained for each standard distance for each $O$. Randomization of distances and starting position (either large or small) was used within each series, and Objective or "physical size" instructions were given (Carlson, 1962).

For the distance estimation experiment, either the smallest or largest distance (far point marked by a stimulus disc) was designated as 100 arbitrary units, and $O$ was required to give verbally the distance from him of each comparison, also marked by a stimulus disc, in respect to the fixed standard. Randomization of comparison distances and disc sizes were employed within each series. Eight estimates for each comparison distance (except equidistance) were obtained with each standard. The order of the three types of judgments were: frontal size, flat size, distance.

The Os were JB and WB, both of whom were experienced in psychophysical observation and both of whom possessed emmetropic vision (corrected for WB).

\section{Frontal Size}

\section{RESULTS}

The data are presented both in metric and angular units to facilitate their use in alternative theoretical analyses. The combined means (in inches) and standard deviations from the two Os are given in Table 1 for the near and far comparison conditions. The results are further separated into ascending and descending series. Separate t-tests were run for each pair of

Table 1. Frontal Size Judgments. Means and standard deviations of frontal-size judgments on ascending and descending trials and for all trials combined. Data are from two observers who each produced eight judgments for each condition, The near comparison distance was 24-in,

the far comparison was at 216-in.

\begin{tabular}{|c|c|c|c|c|c|c|c|c|c|c|c|}
\hline & & \multicolumn{10}{|c|}{ Standard Distance (in.) } \\
\hline & & \multicolumn{2}{|c|}{24} & \multicolumn{2}{|c|}{41.75} & \multicolumn{2}{|c|}{80.25} & \multicolumn{2}{|c|}{164.625} & \multicolumn{2}{|c|}{216} \\
\hline & & asc & desc & osc & desc & asc & desc & asc & $\operatorname{desc}$ & asc & desc \\
\hline & $M$ & 8.00 & 8.00 & 8.00 & 7.95 & 3. 19 & 8.30 & 8.69 & 9.02 & 9.03 & 9.23 \\
\hline Near & $\mathrm{SD}$ & 0 & 0 & .14 & .14 & .14 & .17 & .28 & .30 & .76 & .57 \\
\hline \multirow[t]{3}{*}{ Comp. } & $G M$ & \multicolumn{2}{|c|}{8.00} & \multicolumn{2}{|c|}{7.98} & \multicolumn{2}{|c|}{8.24} & \multicolumn{2}{|c|}{8.85} & \multicolumn{2}{|c|}{9.13} \\
\hline & SD & \multicolumn{2}{|c|}{0} & \multicolumn{2}{|c|}{.14} & \multicolumn{2}{|c|}{.17} & \multicolumn{2}{|c|}{.33} & \multicolumn{2}{|c|}{.68} \\
\hline & $M$ & 5.67 & 5.97 & 6.38 & 6.44 & 6.44 & 6.70 & 7.44 & 7.38 & 8.00 & 8.00 \\
\hline Far & SD & .75 & .77 & .66 & .66 & .64 & .67 & .26 & .28 & 0 & 0 \\
\hline \multirow[t]{2}{*}{ Comp. } & $G M$ & \multicolumn{2}{|c|}{5.82} & \multicolumn{2}{|c|}{6.41} & \multicolumn{2}{|c|}{6.57} & \multicolumn{2}{|c|}{7.41} & \multicolumn{2}{|c|}{8.00} \\
\hline & SD & \multicolumn{2}{|c|}{.78} & \multicolumn{2}{|c|}{.66} & \multicolumn{2}{|c|}{.68} & \multicolumn{2}{|c|}{.28} & \multicolumn{2}{|c|}{0} \\
\hline
\end{tabular}




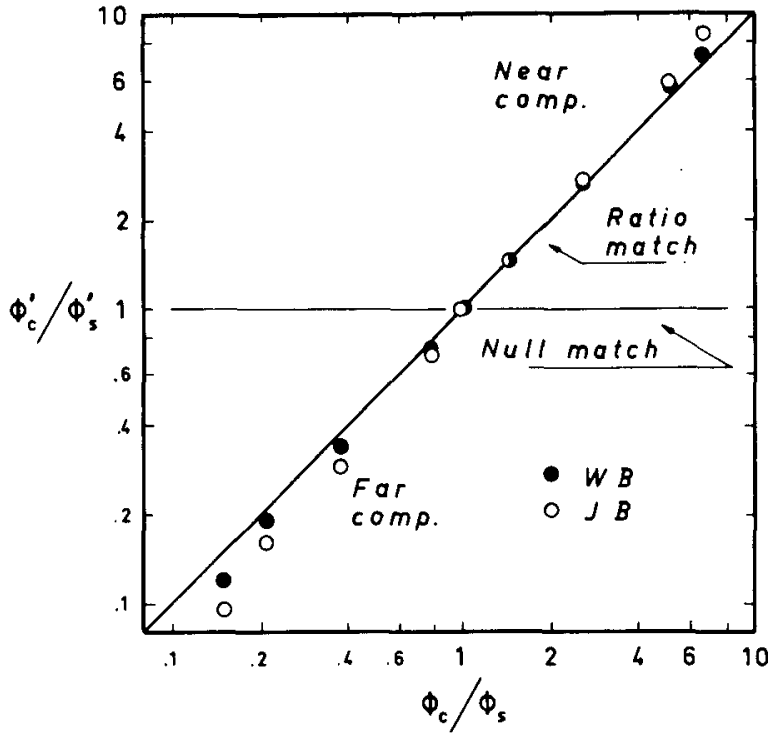

Fig. 1. Frontal-size judgments ( $\varnothing \mathbf{c} / \emptyset^{\prime} \mathbf{s}^{\text {) }}$ as a function of theoretical size $\left(\emptyset \mathrm{c} / \boldsymbol{\sigma}_{\mathrm{s}}\right)$. The theoretical and judged sizes are expressed as ratios involving the visual angles subtended by the comparison and standard stimuli. Mean data are given for two observers (JB \& WB). Coordinates are logarithmic to accord with Equation 2. For more details, see the text

ascending-descending means. Only one test was significant (near comparison, 164.625; $t=2.343$, $\mathrm{df}=14$, $\mathrm{p}<.05)$. The overall trend of the values in Table 1 is toward overconstancy. The near comparison was made too large to match the physical size of the farther standards, and the far comparison was made too small to match closer stimuli. As the distance separating the comparison and standard increased, the overconstancy effect increased.

The individual means were transformed into visual angles subtended at the corneal surfaces in order to apply Equation 1. Computations were done separately for each $O$. The theoretical visual-angle ratio for every distance combination was that value which would exist when the standard and comparison were the same physical size ( 8 in.). This particular value is chosen for convenience only! The ratio is defined as: $\phi_{\mathrm{c}} / \phi_{\mathrm{s}}$, where $\phi_{\mathrm{c}}$ is the visual angle subtended by the comparison and $\phi_{s}$ the angle subtended by the standard. The empirical ratio $\left(\phi^{\prime} \mathrm{c} / \phi_{\mathrm{s}}^{\prime}\right)$ was the visual-angle ratio existing after the completion of a size judgment. In this case $\phi^{\prime}$ ' is an arithmetic mean and $\phi_{\mathrm{s}}^{\prime}$ is equal to $\phi_{\mathrm{s}}$. It is suggested that $\phi_{\mathrm{c}}^{\prime} / \phi_{\mathrm{s}}^{\prime}$ is a power function of $\phi_{\mathrm{c}} / \phi_{\mathrm{s}}$ as described by Equation 1. Data relevant to this hypothesis are shown in Fig. 1. The obtained visual-angle ratios for JB and WB are plotted against the theoretical values. The coordinates are logarithmic to accord with Equation 2 and the trend of points would fall on a straight line if the power function were valid. In this type of plot a ratio of 1.0 represents a null match of visual angles, as indicated on the graph by the horizontal line. This line should describe judgments when the standard and comparison stimuli are at the same distance. Theoretical ratios for the near comparison and far standard are greater than 1.0, while the far-comparison ratios are less than 1.0. A perfect psychophysical match of the theoretical values would fall on the $45^{\circ}$ solid line which has a slope of 1.0. Straight lines with slopes greater than 1.0 indicate overconstancy; less than 1.0, underconstancy. As is plain from Fig. 1, a straight line could fit the individual data points very well, though the deviation from a ratio match is slightly greater for the far comparison than for the near. The direction of these effects are the same for $\mathrm{JB}$ and $\mathrm{WB}$, and they maintain their relative deviations for both comparison conditions. Overconstancy is the rule for all conditions. Because of the near-far effect, and the slight individual differences, straight lines were fit separately to the points obtained from each $O$ and for each comparison distance. The goodness of fit of a least squares solution is excellent. Thus, Equation 1 can be applied to the data, and the appropriate exponents of this equation are given in Table 2 for each condition.

\section{Flat Size}

These data were treated in a manner similar to that described for frontal size. The combined mean judgments and standard deviations are given in Table 3. There was an obvious ascending-descending effect, and t-tests were conducted for each pair of means in Table 3. For the far comparison, differences between ascending and descending means were significant for all distances except the largest. For the near comparison, only the two largest distances were significant ( $t>2.145, \mathrm{df}=14, \mathrm{p}<.05)$. However, contrary to the frontal-size results, the size productions in a longitudinal plane were less than the physical size of the standard. This underconstancy increased with increasing separation between standard and comparison.

Visual-angle ratios were computed to compare theoretical and empirical values. The computed ratios are shown in Fig. 2 for the two Os' mean judgments on both the near and far comparisons. The coordinates are logarithmic with details of interpretation the same as for Fig. 1. Straight lines were fit to these data, and the slopes for all conditions were less than 1.0. The linear fits determined by least squares solutions were excellent. Both Os produced visual-

Table 2. Exponents. Empirical exponents for Equation 1. The values were determined by least squares solutions for data from each of two observers. For details, see the text.

\begin{tabular}{lcccccccc} 
& \multicolumn{3}{c}{ Frontal Size } & \multicolumn{3}{c}{ Flat Size } & \multicolumn{3}{c}{ Distance } \\
& JB & WB & JB & WB & & JB & WB \\
\hline Near Comp. & 1.12 & 1.05 & .94 & .96 & Small St. & 1.01 & 1.02 \\
Far Comp. & 1.21 & 1.10 & .91 & .94 & Large St. & .77 & .65 \\
\hline
\end{tabular}


Table 3. Flat-Size Judgments. Means and standard deviations of flat-size judgments on ascending and descending trials and for all trials combined. Data are from two observers who each produced eight judgments for each condition. The near comparison distance was 24-in, the far comparison was at 182.25-in.

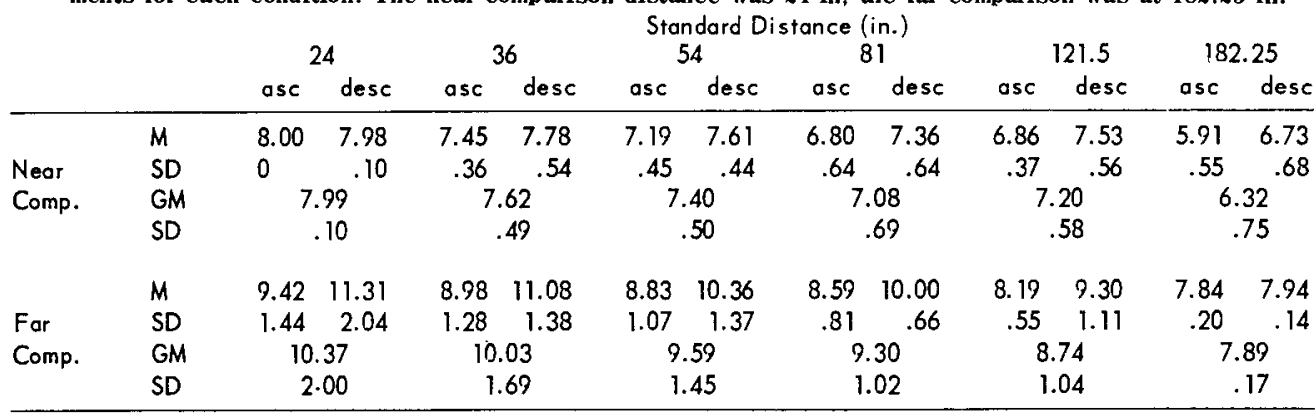

angle ratios which departed from the theoretical values, and the deviations increased as the theoretical ratio departed from 1.0 Equation 1 is valid, and the empirically determined exponents are given in Table 2 for each condition. The near-far effect found for frontal size was not as evident for flat size.

\section{Distance}

The means and standard deviations of the distance estimates are given in Table 4. A different distance series was used for each $O$. It is not clear how these results should be treated to agree with the visualangle analysis described for size productions. There are several alternative approaches with different outcomes. We decided to use visual-angle measures which reflected the ratio task assigned $O$ rather than attempt a more direct connection with the psychophysical method used for size. The theoretical visual-angle ratio was between the subtended angles (at the corneal surface) of the standard and comparison distances as measured in a longitudinal plane from $O$ 's end of the table. When values are computed in this way, the

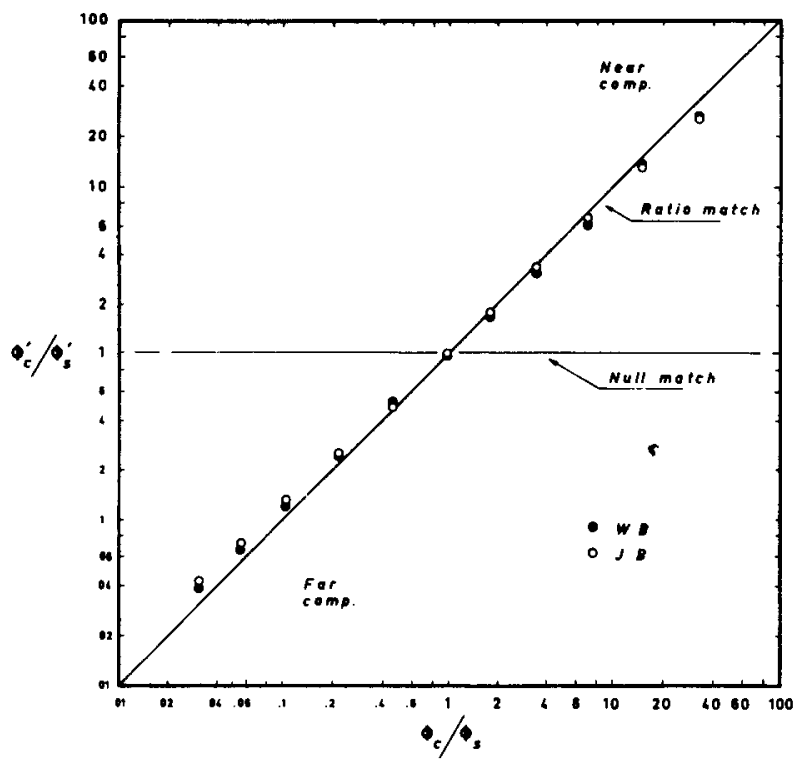

Fig. 2. Flat-size judgments $\left(\sigma^{\prime} \mathrm{c} / \sigma_{\mathrm{s}}^{\prime}\right)$ as a function of theoretical size $(\phi c / \varnothing s)$. For more details, see Fig. 1 and the text. range of ratios sampled is considerably smaller than for either the frontal- or flat-size conditions. The results computed for individual means for both Os are given in Fig. 3 in logarithmic coordinates. In this case $\phi_{\mathrm{s}}$ is the fixed visual angle of the standard, and $\phi_{\mathrm{c}}$ is the visual angle of the comparison. The differences between $O s$ are minor, but there is a marked effect of the standard such that the deviation from a ratio match is more pronounced for the larger standard. The slopes of the best fitting straight lines for the large standard are less than 1.0; whereas, for the small standard the slopes are close to a ratio match. The goodness of fit is satisfactory, but a slight curvilinearity is present over some of the stimulus range. The exponents for Equation 1 also are given in Table 2 .

\section{DISCUSSION}

There are two points we wish to focus upon in discussing the results. The first concerns the quantitative description of the data, and in this regard the adequacy of Equation 1. The second part is more speculative in that we suggest some theoretical connections which probably do and do not exist among the psychophysical functions.

A power function offers a good empirical summary of our results. A straight line can be fit reasonably to the mean points in Figs. 1, 2 and 3; and therefore, Equation 1 is valid. There are differences in the slopes of the linear fits when the near and far comparisons (large and small standards for distance) are treated separately, and the observance of this distinction leads to a more accurate description of the data. This effect is especially present for the distance estimates shown in Fig. 3. Thus, it would be unwise to accept a single exponent as representative of a general function for either size or distance judgments. The experimental method critically affects this value, even when stimulus conditions are practically constant. This also is a problem in the scaling of size judgments at a constant distance (Svenson \& Akesson, 1966). From these facts it does not follow that Equation 1 is invalid (cf., Stevens \& Galanter, 1957). It can be assumed that the basic judgment process underlying the three psychophysical tasks is 
identical, while the exponent depends upon the particular experimental method and stimulus conditions. This conclusion is in agreement with more general statements concerning the importance of the power function in psychophysics (Stevens \& Galanter, 1957), and with the specific view that the power function will be an integral part of a theory of size and distance perception (Akishige, 1961; Gogel, 1964; Ueno, 1962). None of these authors have employed the visual-angle measures we have, though it is still possible to compare our data with previous results. This comparison is favorable in every instance. An exponent greater than 1.0 indicates that an overconstancy effect existed for frontal-size productions (Fig. 1 and Table 2) and this is a common result with Objective instructions (Carlson, 1962; Epstein, 1963; Gilinsky, 1955; Holway \& Boring, 1941; Smith, 1953). From Table 2 the amount of overconstancy can be computed by subtracting the obtained exponents from 1.0. The deviation from size-constancy varies between +.05 and +.21 . These positive values contrast with the deviations for the flat-size condition where underconstancy occurs to a degree which varies between -.04 and -.09 . These findings support data from studies in which the comparison and standard were placed in a longitudinal plane. In experiments of this type the trend is always toward underconstancy (Gilinsky, 1951; Gogel, 1964; Harway, 1963). It also is usually true that underconstancy occurs when distance in a longitudinal plane is estimated directly, though interpretation of previous data is difficult because of differences among comparison methods, and because of the great variability of estimates obtained from groups of observers (Epstein, 1963; Gibson, Bergmann, \& Purdy, 1955; Joynson, Newson, \& May, 1965). In our work the exponent deviated negatively from 1.0 for distance

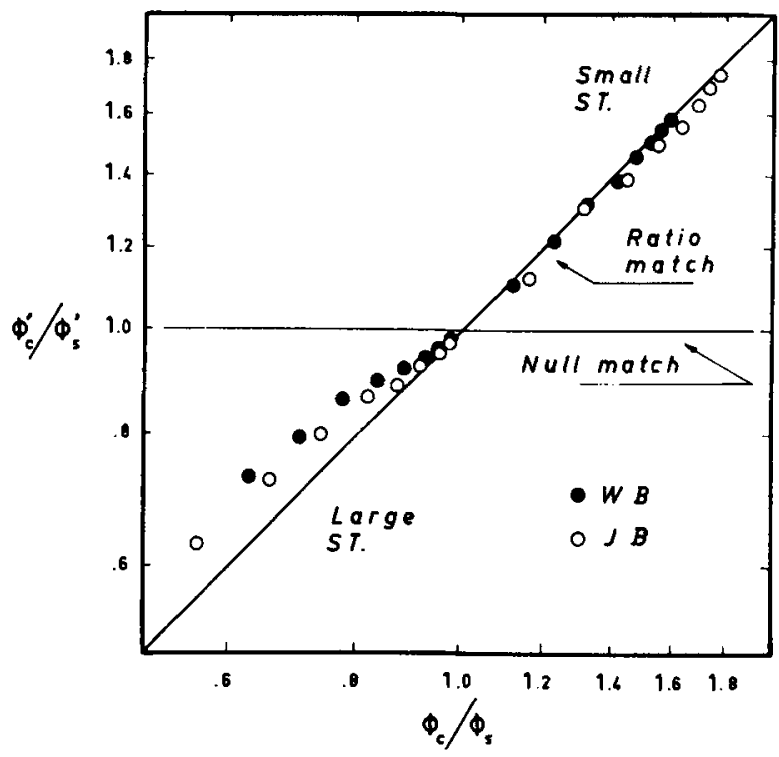

Fig. 3. Distance estimates $\left(\boldsymbol{C}^{\prime} / \boldsymbol{C}^{\prime} \mathbf{s}^{\prime}\right)$ as a function of theoretical distance $(\emptyset \mathrm{c} / \emptyset \mathrm{s})$. For more details, see Fig. 1 and the text.

estimates when the standard was large and covered the entire table top but not when it was small and near to the $O$. The latter slopes were very close to 1.0. It might be possible to derive predictions for these distance estimates from the flat-size results if a few additional assumptions were made.

A quantitative description in terms of Equation 1 is desirable but it leaves unanswered the fundamental question as to what differences among exponents imply for a theory of size and distance perception. In this respect, one thing stands out. Since the exponents differ among the three judgment conditions, frontalsize procuctions should not be considered as dependent

Table 4. Distance Estimates. Means and standard deviations of distance estimates for each of two observers. The small standard was the nearest distance in the comparison series and was called 100 judgment units. The large standard was the farthest distance in the series and also was called 100. Means are expressed in judgment units. The measured actual distances of the stimuli are given both in inches and in judgment units.

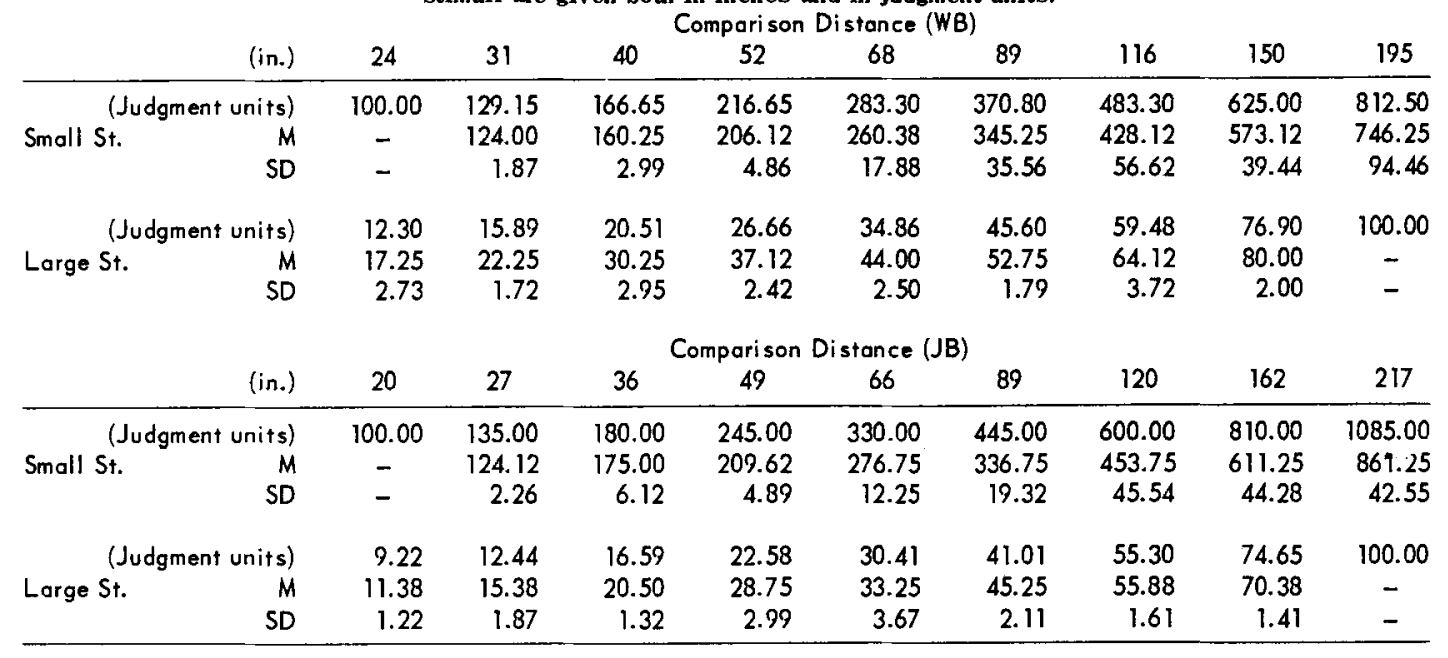


only upon distance estimates. If the exponents for either the distance estimates or the flat-size judgments were the same as for frontal size, one might suggest that overconstancy of frontal size depended upon distance overconstancy (cf., Baird, 1965b; Epstein, Park, \& Casey, 1961). This does not mean that no important relation exists among these judgments, since obviously the three types are correlated. In order to obtain more accurate predictions of size judgments from distance judgments, or vice versa, we may have to define the stimulus situation in more detail. For example, if the estimation of distance ratios between the comparison and standard sizes was constant in both the frontal and flat conditions, the orientation of the size stimulus was the factor accompanying the change in exponent. The visual-angle ratios which os were required to produce under the frontal and flat conditions were not the same. The exponent varied systematically with stimulus orientation and hence with the theoretical visualangle ratio to be produced. It is not known whether the exponents in Table 2 are linked with the stimulus orientation in respect to the longitudinal plane (the table), to Os' lines of sight, or to a combination of these two variables.

Alternative interpretations of our results can be attempted, and one which has gained attention lately is the "response-bias" hypothesis. According to Carlson (1962) and Carlson and Tassone (1962), the overconstancy found with Objective instructions is due to the fact that os overcompensate in their relative-size productions for differences in relative distance between the comparison and standard stimulus. The nearer stimulus must look larger in size than the more distant, and this compensation increases in proportion to the distances separating the standard and comparison. Although this may be an accurate description of the judgment process for frontal-size, it does not account for the flat-size data. Here, underconstancy was obtained even though the instructions were Objective. Wohlwill $(1964,1965)$ also has suggested that a response bias is operating when a longitudinal extent (distance) is bisected. His results and those of others (Denis-Prinzhorn, 1960; Gruber, 1954) show that in bisection the near half of an interval is made too large (physically) to match the far half. However, overconstancy occurs only for this limited situation. Gogel (1964) reports this effect for close distances but when larger separations between comparison and standard were used, underconstancy was the rule. We did not find any pronounced deviation from an accurate match of flat size when both stimuli were near each other in distance. As the ratio between stimulus distances increased, there was a clear underconstancy. This effect also existed for distance estimates involving the large standard.

\section{References}

Akishige, Y. Theoretical problems on the structure of perceptual constancies. In Y. Akishige (Ed.), Kyushu Psychological Studies. Bull. Fac. Lit. Kyushu University, 1961, No. 7. Pp. 1-57.

Baird, J. C. Stimulus and response factors in size instruction effects. Percept. mot. Skills, 1965a, 21, 915-924.

Baird, J. C. Area and distance estimation of single and multiple stimuli. Vis. Res., 1965b, 5, 661-678.

Carlson, V. R. Size-constancy judgments and perceptual compromise. J. exp. Psychol., 1962, 63, 68-73.

Carlson, V. R., \& Tassone, E. P. A verbal measure of the perspective attitude. Amer. J. Psychol., 1962, 75, 644-647.

Denis-Prinzhom, M. Perception des distances et constance des grandeurs (étude génétique). Arch. Psychol., Genève, 1960, 37, 181-309.

Epstein, W. Attitudes of judgment and the size-distance invariance hypothesis. J. exp. Psychol., 1963, 76, 257-265.

Epstein, W., Park, J., \& Casey, A. The current status of the sizedistance hypotheses. Psychol. Bull., 1961, 58, 491-514.

Gibson, E. J., Bergman, R., \& Purdy, J. The effect of prior training with a scale of distance on absolute and relative judgments of distance over ground. J. exp. Psychol., 1955, 50, 97-105.

Gibson, J. J. The perception of the visual world. Houghton Mifflin: Boston, 1950.

Gilinsky, A. S. Perceived size and distance in visual space. Psychol. Rev., 1951, 58, 460-482.

Gilinsky, A. S. The effect of attitude upon the perception of size. Amer. J. Psychol., 1955, 68, 173-192.

Gogel, W. C. Visual perception of spatial extent, J. opt. sac. Amer., 1964, 54, 411-416.

Gruber, H. E. The relation of perceived size to perceived distance. Amet. J. Psychol., 1954, 67, 411-426.

Harway, N. I, Judgment of distance in children and adults. $J$. exp. Psychol., 1963, 65, 385-390.

Holway, A. H., \& Boring, E. G. Determinants of apparent visual size with distance variant. Amer. J. Psychol., 1941, 54, 21-37.

Joynson, R. B., Newson, L. J., \& May, D. S. The limits of overconstancy. Quart. J. exp. Psychol., 1965, 17, 209-216.

Stevens, S. S., \& Galanter, E. H. Ratio scales and category scales for a dozen perceptual continua. J. exp. Psychol., 1957, 54 $377-411$.

Stevens, S. S., \& Guirao, M. Subjective scaling of length and area and the matching of length to loudness and brightness. $J$. exp. Psychol., 1963, 66, 177-186.

Smith, W. M. A methodological study of size-distance perception. J. Psychol., 1953, 35, 143-153.

Svenson, 0., \& Akesson, C. A. Fractional and multiple estimates in ratio scaling. Rep. Psychol. Lab., University Stockholm, No. $202,1966$.

Ueno, $T$. The size-distance invariance hypothesis and the psychophysical law. Jap. Psychol. Res., 1962, 4, 99-112.

Wohlwill, J. F. Changes in distance judgments as a function of corrected and noncorrected practice. Percept. mot. Skills, 1964, $19,403-413$.

Wohl will, J. F. Texture of the stimulus field and age as variables in the perception of relative distance in photographic slides. J. exp. Child Psychol., 1965, 2, 163-177.

\section{Note}

1. This research was conducted at Walter Reed and was prepared for publication while J. B. was at the University of Stockholm. The preparation of the manuscript was supported (in part) by a Public Health Service fellow ship (Number 1-F2-20, 952-01) from the National Institute of Mental Health, Public Health Service.

(Accepted for publication February 6, 1967.) 